\title{
Competitividade inter-regional de sistemas de produção de bovinocultura de corte
}

\author{
Interregional competitiveness of the beef cattle production system
}

\section{Ricardo Pedroso Oaigen ${ }^{\text {I* }}$ Júlio Otávio Jardim Barcellos ${ }^{\text {II }}$ Maria Eugênia Andrighetto Canozzi ${ }^{\mathrm{II}}$ Jean Carlos dos Reis Soares ${ }^{\mathrm{II}}$ Leonardo Canali Canellas $^{\mathrm{II}}$ Carine Oliveira Alves $^{\mathrm{I}}$ Heliton Ribeiro Tavares ${ }^{\text {II }}$ Fabricio Martins da Costa ${ }^{\text {IV }}$}

RESUMO

O trabalho apresenta uma metodologia de avaliação de sistemas de produção através da mensuração da competitividade interna na bovinocultura de corte. Durante o primeiro trimestre de 2010, foram aplicados 65 questionários com pecuaristas, sendo 36 entrevistas na Região Sul (Estado do Rio Grande do Sul) e 29 na Região Norte (Estados do Pará e Rondônia). Foram definidos os principais direcionadores que afetam a competitividade interna tecnologia, gestão, relações de mercado e ambiente institucional, sendo atribuido um peso especifico para cada direcionador, a fim de obter o índice de competitividade. Os resultados foram analisados estatisticamente pela teoria de resposta ao item e pela análise de correspondência (ANACOR) com o software SPSS ${ }^{\circledast}$. A Região Sul apresentou uma maior competitividade que a Região Norte. Independente da região, os fatores críticos de competitividade foram: integração lavoura-pecuária, planejamento estratégico, cálculo de indicadores financeiros, formação de preços, acesso a inovações tecnológicas e organização dos produtores.

Palavras-chave: agronegócio, bovinocultura, cadeia produtiva, competitividade, sistemas de produção.

\section{ABSTRACT}

This paper presents a methodology for evaluating production systems by measuring the intern competitiveness of in beef cattle. During the first quarter of 2010, questionnaires were administered to 65 farmers, 36 interviews in the Southern Region (State of Rio Grande do Sul) and 29 in the Northern Region (States of Pará and Rondonia). Defined the key drivers that affect the international competitiveness - technology, management, market relations and institutional environment, an specific weight was assigned to each in order to get the index of competitiveness. The results were statistically analyzed by the item response theory and the correspondence analysis (ANACOR) with the SPSS ${ }^{\circledR}$ software.
The South was more competitive than the North. Regardless of region, the critical factors of competitiveness were: crop-livestock integration, strategic planning, calculation of financial indicators, beef pricing, access to technological innovations and organization offarmers.

Key words: agribusiness, beef cattle, productive chain, competitiveness, production system.

\section{INTRODUÇÃO}

A bovinocultura de corte brasileira tem passado por mudanças estruturais e conjunturais, sobretudo no uso de tecnologias agropecuárias, no aumento das exportações de carne e na distribuição geográfica do rebanho. Essas transformações permitiram expansões significativas do rebanho para as Regiões Centro-Oeste e Norte, devido à competição por áreas para agricultura no Sudeste e Sul do Brasil. Dentro desse contexto, estudos que mensurem a competitividade de sistemas agroindustriais passaram a ser fundamentais (VAN DUREN et al., 1991; SILVA \& BATALHA, 1999). Dentre as definições de competitividade, a que melhor se aplica é como sendo a capacidade sustentável de sobreviver e, de preferência, crescer nos mercados concorrentes ou em novos mercados, através de um sistema de informações capaz de suprir as necessidades gerenciais derivadas do planejamento

\footnotetext{
IFaculdade de Medicina Veterinária, Universidade Federal do Pará (UFPA), Avenida Universitária S/N, Bairro Jaderlândia, 68745-000, Castanhal, PA, Brasil. E-mail: oaigenricardo@ufpa.br.*Autor para correspondência.

IIDepartamento de Zootecnia, Faculdade de Agronomia, Universidade Federal do Rio Grande do Sul (UFRGS), Porto Alegre, RS, Brasil. IIIInstituto de Ciências Exatas e Naturais, Universidade Federal do Pará (UFPA), Belém, PA, Brasil.

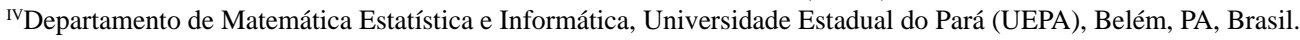
Recebido 23.01.13 Aprovado 19.03.13 Devolvido pelo autor 10.06.13 CR-2013-0092
} 
de longo prazo (CALLADO \& MORAES FILHO, 2008). Para isso, a sua determinação pode ser realizada com o uso de fatores sistêmicos (ambiente concorrencial), estruturais (relativos ao mercado) e internos (relativos à empresa) (SILVA, 2004).

A formulação e a implementação de estratégias são influenciadas por fatores externos e internos às empresas. Os fatores empresariais (internos) são aqueles em que a empresa tem poder de decisão e referem-se ao estoque de recursos acumulados pela empresa; os estruturais (internos e/ou externos) são aqueles em que a capacidade de intervenção da empresa é limitada; e, por fim, os sistêmicos (externos) constituem externalidades strictu sensu para a empresa, a qual detém escassa ou nenhuma possibilidade de intervir (KUPFER \& HASENCLEVER, 2002).

Nesse sentido, este estudo objetiva propor e validar uma metodologia que mensure e compare a competitividade interna da bovinocultura de corte em diferentes regiões, a fim de evidenciar ações prioritárias que devem ser tomadas para maximizar as vantagens competitivas regionais, uma vez que as pesquisas anteriores são genéricas, no sentido de englobar toda a cadeia produtiva. Esse tipo de análise permitirá suprir uma lacuna de métodos de aferição da eficiência dos sistemas de produção pecuários, que permita comparar diferentes sistemas produtivos. Além disso, poderá, no futuro, ser adaptada para outras cadeias produtivas na produção animal.

\section{MATERIAL E MÉTODOS}

O trabalho teve início com um roteiro de entrevistas realizadas com pecuaristas, durante o primeiro trimestre de 2010, nas Regiões Norte (29 propriedades nos Estados do Pará e Rondônia) e Sul (36 propriedades no Estado do Rio Grande do Sul), totalizando 65 produtores. A metodologia aplicada foi baseada numa síntese de procedimentos utilizados em diagnósticos e modelos conceituais de cadeias produtivas, cujo foco era o estudo da competitividade da cadeia produtiva da carne bovina (SILVA \& BATALHA, 1999; IEL/CNA/SEBRAE, 2000; BATALHA, 2007; FAMATO/FABOV, 2007).

A pesquisa em questão é específica para os sistemas de produção, sendo este um avanço e/ou inovação proposto pelo presente estudo, referenciada por trabalhos que utilizaram o enfoque sistêmico do produto (commodity system approach) e o gerenciamento da cadeia de suprimentos (supply chain management) para análise de cadeias produtivas agroindustriais (SILVA \& BATALHA, 1999; IEL/
CNA/SEBRAE, 2000; SENAR/SEBRAE/FARSUL, 2005; BATALHA, 2007; FAMATO/FABOV, 2007).

Inicialmente, foram definidos os principais direcionadores que afetam a competitividade interna, sendo: tecnologia (TE), gestão (GE), relações de mercado (RM) e ambiente institucional (AI) (SILVA \& BATALHA, 1999). A partir da definição dos direcionadores, houve um desdobramento em 31 fatores (Tabela 1). Os direcionadores TE, GE, RM e AI foram constituídos por dez, dez, quatro e sete

Tabela 1 - Direcionadores e fatores, com seus respectivos pesos, utilizados para mensurar a competitividade "dentro da porteira" na bovinocultura de corte.

\begin{tabular}{|c|c|}
\hline Direcionador & Peso relativo \\
\hline Tecnologia & 3,50 \\
\hline I1. Adequação de um sistema produtivo & 0,10 \\
\hline I2. Qualidade, manejo e espécies de pastagens & 0,15 \\
\hline I3. Suplementação animal & 0,15 \\
\hline I4. Integração lavoura e pecuária & 0,10 \\
\hline I5. Manejo reprodutivo & 0,15 \\
\hline I6. Genética do rebanho & 0,05 \\
\hline I7. Sanidade do rebanho & 0,10 \\
\hline I8. Controle zootécnico & 0,05 \\
\hline I9. Assessoria técnica regular & 0,10 \\
\hline I10. Manejo de rotina com os animais & 0,05 \\
\hline Subtotal & 1,00 \\
\hline Gestão & 3,50 \\
\hline I1. Capacitação da mão de obra & 0,15 \\
\hline I2. Patrimônio & 0,05 \\
\hline I3. Orçamentação e fluxo de caixa & 0,10 \\
\hline I4. Planejamento estratégico & 0,05 \\
\hline I5. Controle dos custos de produção & 0,15 \\
\hline I6. Cálculo de indicadores financeiros & 0,10 \\
\hline I7. Identificação do rebanho & 0,10 \\
\hline I8. Comercialização & 0,10 \\
\hline I9. Informatização da propriedade & 0,05 \\
\hline I10. Escala de produção & 0,15 \\
\hline Subtotal & 1,00 \\
\hline Relações de mercado & 2,00 \\
\hline I1. Relação produtor-fornecedor & 0,35 \\
\hline I2. Relação produtor-frigorífico & 0,35 \\
\hline I3. Formação de preços & 0,15 \\
\hline I4. Diferenciação de produtos & 0,15 \\
\hline Subtotal & 1,00 \\
\hline Ambiente institucional & 1,00 \\
\hline I1. Acesso a inovações tecnológicas & 0,15 \\
\hline I2. Política e fiscalização tributária e trabalhista & 0,15 \\
\hline I3. Política e fiscalização ambiental & 0,15 \\
\hline I4. Política de crédito agropecuário & 0,10 \\
\hline I5. Política e fiscalização sanitária & 0,15 \\
\hline I6. Legislação oficial e regularização fundiária & 0,10 \\
\hline I7. Organização dos produtores & 0,20 \\
\hline Subtotal & 1,00 \\
\hline
\end{tabular}


fatores, respectivamente, sendo atribuído um peso específico para cada um deles, a fim de determinar o índice de competitividade (IC).

O questionário apresentava quatro perguntas para cada fator. As respostas poderiam ser positivas ou negativas. Quanto maior o número de respostas positivas, maior o grau de competitividade do fator, do respectivo direcionador e, consequentemente, do IC. Atribuíram-se, ainda, pesos diferenciados aos fatores, em virtude do grau de importância para o direcionador. Com esse procedimento, a avaliação final dos direcionadores foi dada pela equação 1 :

$$
N D_{i}=\sum_{j=1}^{n} \frac{N F_{j}}{P F_{j}} x P D_{i}
$$

em que $\mathrm{ND}_{\mathrm{i}}$ é avaliação final do direcionador $\mathrm{i} ; \mathrm{NF}_{\mathrm{j}}$ é a nota dada ao fator $\mathrm{j} ; \mathrm{PF}_{\mathrm{j}}$ é o peso atribuído ao fator $\mathrm{j} ; \mathrm{PD}_{\mathrm{i}}$ é o peso atribuído ao direcionador i.

As notas dos fatores $\left(\mathrm{NF}_{\mathrm{j}}\right)$ foram obtidas a partir das respostas dos entrevistados (equação 2). O percentual de acerto $\left(\mathrm{PA}_{\mathrm{j}}\right)$ de cada resposta e o peso $\left(\mathrm{PF}_{\mathrm{j}}\right)$ atribuído determinaram a nota de cada fator.

$$
N F_{j}=\sum_{j=1}^{n} P A_{j} P F_{j}
$$

Os fatores foram avaliados a partir da quantidade de respostas positivas, utilizando a escala Likert. O critério utilizado para qualificar a resposta e determinar o percentual de acertos foi: MF- muito favorável: quatro respostas positivas (100\%); Ffavorável: três respostas positivas $(75 \%) ; \mathrm{N}-$ neutro: duas respostas positivas (50\%); D- desfavorável: uma resposta positiva $(25 \%)$; MD- muito desfavorável: nenhuma resposta positiva $(0 \%)$.

Posteriormente, foi originada uma nova equação (equação 3) para calcular o IC de cada sistema de produção e posterior mensuração da média aritmética da amostra.

$$
\mathrm{ND}=\mathrm{ND}_{\text {tecnologia }}+\mathrm{ND}_{\text {gestão }}+\mathrm{ND}_{\text {relações de mercado }}+
$$

e gestão contribuíram, cada um, com 3,5; relações de mercado, com 2,0; e ambiente institucional, com 1,0. Os direcionadores TE e GE, os quais dependem em maior grau da ação do pecuarista, da sua atitude empresarial e do nível de controle, receberam maiores pesos, sendo definidos como fatores internos de competitividade, enquanto que os demais, RM e AI, foram definidos com fatores externos (KUPFER \& HASENCLEVER, 2002). A classificação final do IC foi obtida a partir do seguinte critério: $\mathrm{MF}-\mathrm{IC}>8,0 ; \quad \mathrm{F}-6,0<\mathrm{IC} \leq 8,0 ; \quad \mathrm{N}-4,0<\mathrm{IC} \leq 6,0$; D-2,0<IC $\leq 4,0 ; \mathrm{MD}-\mathrm{IC} \leq 2,0$.
O tratamento estatístico dos resultados envolveu a utilização da teoria de resposta ao item (TRI), por permitir comparar populações diferentes que foram submetidas ao mesmo tipo de avaliação (ANDRADE et al., 2000). Também foi utilizada a análise de correspondência (ANACOR) para identificação de associações entre os direcionadores e fatores com o respectivo grau de competitividade, permitindo representar graficamente a natureza das relações existentes entre as variáveis. Foi necessária a realização do teste do qui-quadrado para avaliação da dependência das observações para um $\mathrm{P}<0,05$ (MANGABEIRA et al., 2002).

\section{RESULTADOS}

Os sistemas de produção podem ser caracterizados como grandes propriedades, pois $80 \%$ possuíam mais de 900 hectares. As principais atividades, em parceria com a bovinocultura de corte, foram as lavouras de arroz e/ou soja na Região Sul e a bovinocultura leiteira no Norte. O grau de instrução do pecuarista foi superior no Sul, uma vez que $70 \%$ possuíam graduação, $18 \%$ pós-graduação e $12 \%$ ensino médio, enquanto que no Norte $31 \%$ possuíam graduação, $10 \%$ pós-graduação, $38 \%$ ensino médio e $21 \%$ ensino fundamental. Nas duas regiões, a imensa maioria (92\%) dos produtores trabalha, exclusivamente, na propriedade rural.

O valor do IC médio foi de 7,31 (F) e 5,32

$(\mathrm{N})$ e, com relação aos direcionadores, os valores foram para TE 7,88 (F) e 6,02 (N), GE 6,65 (F) e $5,11(\mathrm{~N})$, RM 6,06 (F) e 5,12 (N) e AI 6,51 (F) e 4,96 (N) para as Regiões Sul e Norte, respectivamente. As análises de correspondência dos 31 fatores, em seus respectivos direcionadores, para as duas regiões analisadas, estão ilustradas na figura 1. É importante destacar que cada fator é representado pelo caractere "I", seguido de sua respectiva numeração, de acordo com a tabela 1 .

No direcionador tecnologia, a integração lavoura pecuária (I4) foi associada com o grau de competitividade MD, sobretudo na Região Norte. Na Região Sul, o fator I4 também apresentou uma maior proximidade com o grau MD, pois $10 \%$ dos sistemas de produção foram classificados com um menor grau de competitividade para esse item.

Em relação à gestão técnico-financeira, a realidade mostrou-se distinta nas duas regiões. Enquanto que na Região Sul o índice de competitividade apresentou um grau $\mathrm{F}$, na Região Norte a realidade é crítica, visto que $24,1 \%$ dos sistemas de produção foram classificados como D. 
(a)
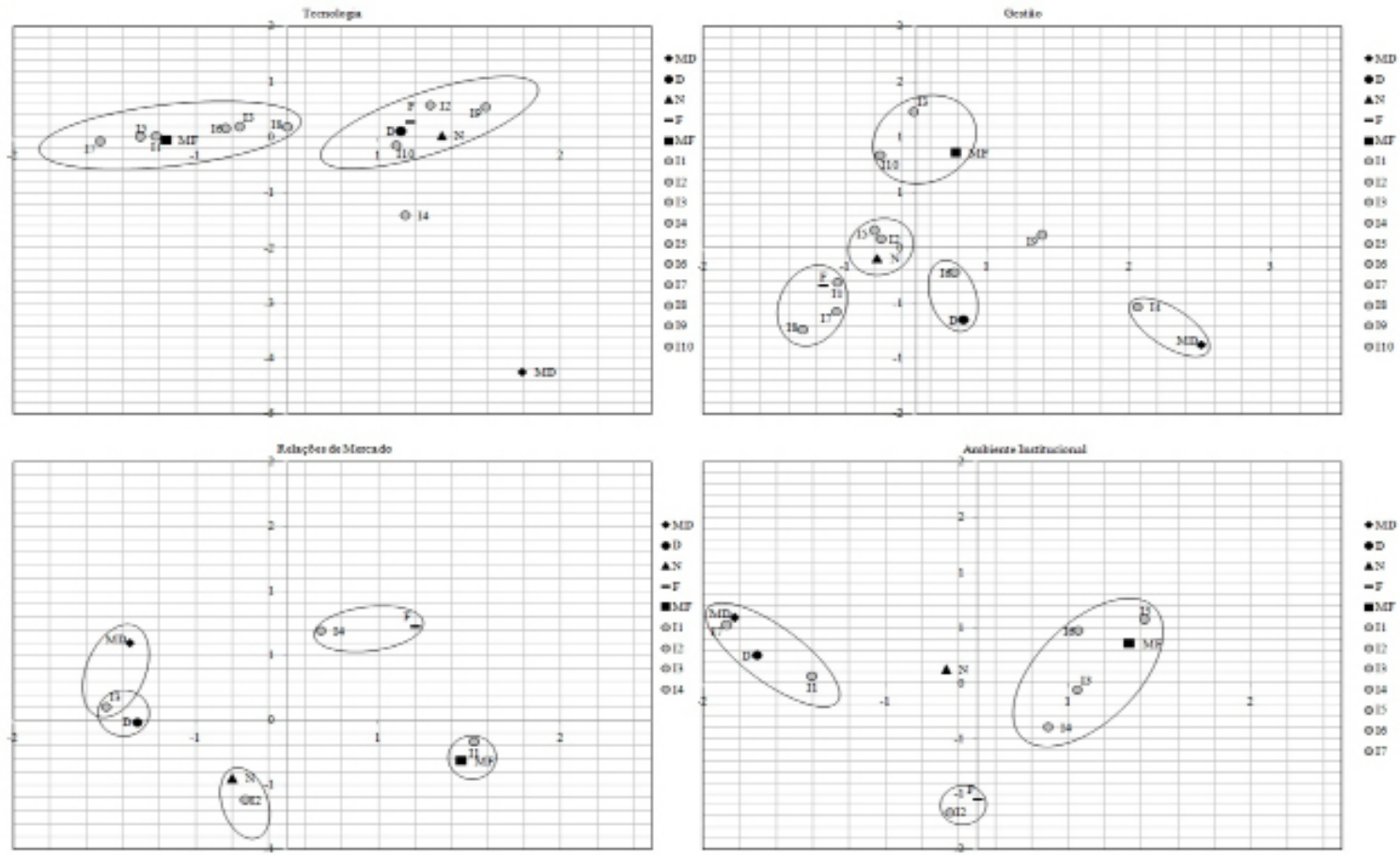

(b)
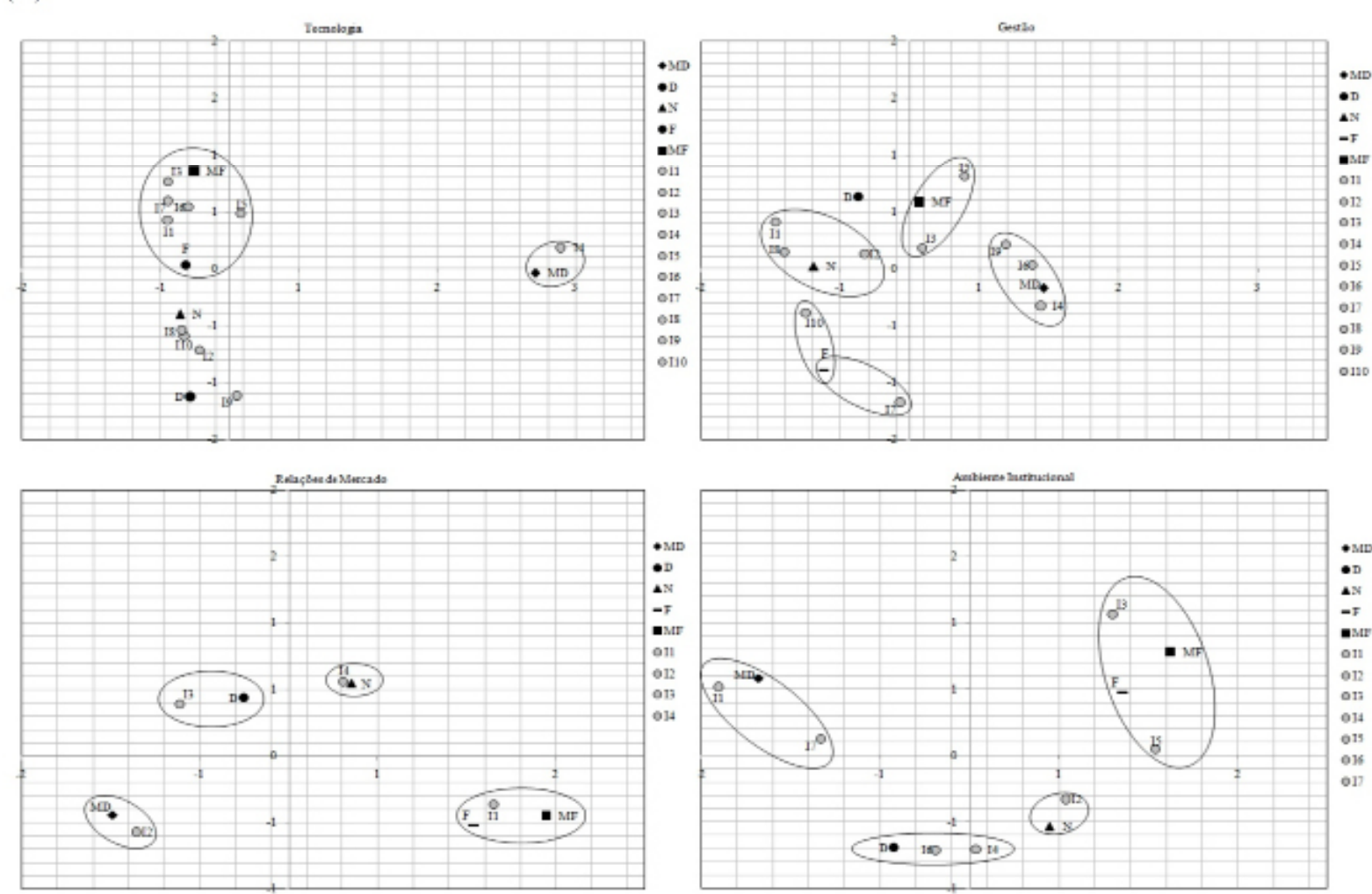

Figura 1 - Análise de correspondência dos fatores, dentro do seu respectivo direcionador, em sistemas de produção de bovinocultura de corte nas regiões Sul (a) e Norte (b) do Brasil. MD: muito desfavorável; D: desfavorável; N: neutro; F: favorável; MF: muito favorável. 
Um fator de competitividade da gestão que merece uma atenção especial é o planejamento estratégico (I4): nas duas regiões esse foi um fator que apresentou relação com o grau MD. Os fatores informatização (I9/GE) e cálculo de indicadores financeiros (I6/GE) também ficaram aquém do desejado em ambas as regiões.

Referente ao direcionador relações de mercado, a relação produtor-fornecedor (I1/RM) foi classificada positivamente nas Regiões Norte e Sul. O fator diferenciação de produtos (I4/RM) foi considerado favorável na Região Sul e neutro da Região Norte. Os demais fatores, relação produtorfrigorífico (I2/RM) e formação de preços (I3/RM), apresentaram um desempenho negativo em ambas as regiões, sobretudo I3, que apresentou uma associação com o grau de competitividade MD e D na Região Sul.

Por fim, no ambiente institucional, os fatores críticos nas duas regiões foram o acesso a inovações tecnológicas (I1) e a organização dos produtores rurais (I7). Existe uma associação entre o grau de competitividade D e a legislação oficial e regularização fundiária (I6/AI) e a política de crédito agropecuário (I4/AI), ao contrário da Região Sul, que apresentou grau de competitividade MF para esses mesmos fatores.

\section{DISCUSSÃO}

A maior integração com a agricultura relatada para a Região Sul é um importante fator de competitividade, pois proporciona a maximização do uso de recursos para o aumento da produtividade e rentabilidade dos sistemas de produção. Essa situação de maior competitividade é devido a uma maior especialização, até mesmo pela proximidade de centros de desenvolvimento e difusão do conhecimento. No entanto, a conjuntura atual mostra que a bovinocultura de corte no Sul do país vem perdendo espaço para outras atividades (lavouras agrícolas e reflorestamento), consequência da maior rentabilidade dessas.

Na Região Norte, ocorreu uma colonização recente há, aproximadamente, quatro décadas, por imigrantes que vieram de outras regiões do país. A pecuária amazônica evoluiu pelos campos e cerrados, sendo inicialmente destinadas à criação de gado bovino para consumo regional (CEPEA, 2002). Posteriormente, houve um acentuado crescimento do uso de pastos cultivados, formados por gramíneas bem adaptadas, principalmente as espécies de Braquiária spp.

O aumento do uso de tecnologias de produção, em ambas as regiões, está relacionado aos trabalhos desenvolvidos em instituições de pesquisa e ensino em áreas de genética, sanidade, reprodução animal, nutrição e manejo de pastagens tropicais e subtropicais (EUCLIDES FILHO, 2007). Apesar do aumento na produção de carne e na produtividade dos sistemas de produção, o potencial dessa atividade está muito aquém do real.

O pecuarista necessita aperfeiçoar sua capacidade de gestor do negócio, já que a grande maioria das empresas rurais desconhece seu custo de produção ou não mensura seus indicadores técnicofinanceiros (OAIGEN \& BARCELLOS, 2008). O gerenciamento da informação, objetivando a tomada de decisões precisa, maximiza o aproveitamento dos recursos disponíveis e a lucratividade da atividade. A questão do desconhecimento de custos de produção foi constatação recorrente com agentes da cadeia, assim como falta de planejamento, baixa adequação da mão de obra das propriedades, tanto em nível operacional como gerencial, e deficiências nos controles zootécnicos dos rebanhos (IEL/CNA/ SEBRAE, 2000).

Em relação ao ambiente organizacional interno das empresas, o planejamento estratégico deve ser baseado em três fatores: gestão (estratégia do negócio, estratégia de operações e foco gerencial), tecnologia (produto, processo e informação) e pessoas (qualificação, liderança, conhecimento, aprendizado e cultura) (DI SERIO \& VASCONCELLOS, 2008). Para isso, é fundamental a existência de pessoas capacitadas e que saibam utilizar os recursos provenientes dessa inovação (internet, softwares, planilhas, etc.).

A busca por uma maior coordenação da cadeia produtiva da carne bovina é uma das metas principais dos órgãos públicos e privados. Entretanto, no mercado da carne bovina, predominam estruturas oligopolísticas a montante da produção agropecuária e oligopsônicas a jusante (ARAÚJO, 2005), ou seja, os pecuaristas rurais são tomadores de preços, mas não conseguem formar preços para seus produtos. Além disso, essa cadeia é caracterizada pela assimetria de informações entre os elos, o que, consequentemente, traz conflitos e relações oportunistas entre seus atores, principalmente, entre frigoríficos e produtores rurais (MALAFAIA et al., 2009).

A avaliação do impacto do ambiente externo sobre os sistemas de produção foi desfavorável para as questões envolvendo tributação excessiva, falhas na política sanitária e condições precárias de financiamento (crédito agropecuário). Outras áreas que merecem atenção devido ao impacto negativo referemse às condições macroeconômicas, informações 
estatísticas, coordenação dos agentes da cadeia e comércio exterior (IEL/CNA/SEBRAE, 2000).

Em relação ao fator I1/AI, essa situação crítica pode estar associada, em parte, ao desinteresse e à falta de comprometimento dos pecuaristas em relação aos trabalhos gerados nas universidades e centros de pesquisa, comprometendo a efetiva difusão do conhecimento e das tecnologias agropecuárias. Diante disso, ações devem ser desenvolvidas pelos órgãos de fomento a pesquisa e extensão rural, sejam públicos ou privados, visando a aproximar o pecuarista das inovações tecnológicas.

A questão fundiária na região amazônica merece atenção, visto que boa parte das propriedades rurais não possui escritura pública ou título definitivo (HOMMA, 2010). Essa situação impossibilita aos pecuaristas acesso ao crédito agropecuário, pois há necessidade do cadastro ambiental rural (CAR) e do licenciamento ambiental rural (LAR). Outro fator responsável pela perda da competitividade interna é a falta de organização dos produtores rurais, já que são poucos exemplos de produtores que efetivamente exercem cooperação e alianças estratégicas com outros elos da cadeia, ou mesmo entre eles (SAAB et al., 2009).

\section{CONCLUSÃO}

Os sistemas de produção de bovinos de corte na Região Sul do Brasil apresentaram uma maior competitividade em relação aos localizados na Região Norte, consequência da maior especialização da atividade e capacitação empresarial. Aspectos tecnológicos relacionados com a produção animal apresentaram um nível de competitividade satisfatório em ambas as regiões. Avanços são necessários, principalmente, no gerenciamento de tecnologias, no uso de ferramentas de gestão, nas relações entre os agentes da cadeia produtiva, na organização dos produtores e no acesso a inovações tecnológicas (extensão rural).

Deve-se considerar que a bovinocultura de corte é uma atividade heterogênea nas diferentes regiões brasileiras e, apesar das limitações de amostragem e metodologia, buscou-se identificar os aspectos principais que afetam a competitividade interna, seja na Região Sul ou Norte do país. Contudo, independentemente da região avaliada e/ou do objeto de estudo, o aperfeiçoamento e o desenvolvimento de novas pesquisas que mensurem a competitividade de sistemas de produção são necessários, para que sirvam como subsídios aos órgãos públicos e privados no desenvolvimento de ações articuladas entre os elos que compõem a cadeia produtiva da carne bovina.

\section{COMITÊ DE ÉTICA E BIOSSEGURANÇA}

Atesto que o manuscrito intitulado "Competitividade inter-regional de sistemas de produção de bovinocultura de corte", submetido a Revista Ciência Rural, foi aprovado no Programa de Pós-Graduação em Zootecnia da Universidade Federal do Rio Grande do Sul e seguiu todas as Normas e Preceitos Legais no que se refere às questões de Ética e Pesquisa com Humanos.

\section{REFERÊNCIAS}

ANDRADE, D.F et al. Teoria da resposta ao item: conceitos e aplicações. Campinas: SINAPE, 2000. 164p.

ARAÚJO, M.J. Fundamentos de Agronegócio. 2.ed. São Paulo: Atlas, 2005. 160p.

BATALHA, M.O. Gestão agroindustrial. 3.ed. São Paulo: Atlas, 2007. 778p.

CALLADO, A.A.C.; MORAES FILHO, R.A. Gestão empresarial no agronegócio. In: (Org.). Agronegócio. 2.ed. São Paulo: Atlas, 2008. p. $\overline{20-29 .}$

CEPEA(CENTRO DEESTUDOS AVANÇADOS EMECONOMIA APLICADA). Economia da pecuária de corte na região norte do Brasil. Piracicaba: CEPEA/ESALQ/USP, 2002. 77p.

DI SERIO, L.C.; VASCONCELLOS, M.A. Estratégia e competitividade empresarial - inovação e criação de valor. São Paulo: Saraiva, 2008. 364p.

EUCLIDES FILHO, K. Bovinocultura de corte no Brasil. Revista de Política Agrícola, v.26, n.4, p.121-128, 2007. Disponível em: <http://www.embrapa.br/publicacoes/tecnico/revistaAgricola/rpade-2007/RPA\%204-2007ultima.pdf>. Acesso em: 15 mar. 2011.

FAMATO/FABOV (FEDERAÇÃO DA AGRICULTURA E PECUÁRIA DO ESTADO DE MATO GROSSO / FUNDO DE APOIO A BOVINOCULTURA DE CORTE). Diagnóstico da cadeia produtiva agroindustrial da bovinocultura de corte do Estado de Mato Grosso. Cuiabá: FAMATO/FABOV, 2007. 522p.

HOMMA, A. Política agrícola ou política ambiental para resolver os problemas da Amazônia. Revista de Política Agrícola, v.29, n.1, p.99-102, 2010. Disponível em: <http://www.embrapa. br/publicacoes/tecnico/revistaAgricola/rpa-2010/Revista_Pol_ Agricola_01_2010.pdf >. Acesso em: 8 out. 2010.

IEL/CNA/SEBRAE (INSTITUTO EUVALDO LODI / CONFEDERAÇÃO NACIONAL DA AGRICULTURA / SERVIÇO BRASILEIRO DE APOIO ÀS MICRO E PEQUENAS EMPRESAS). Estudo sobre a eficiência econômica e competitividade da cadeia agroindustrial da pecuária de corte no Brasil. Brasília, 2000. 398p.

KUPFER D.; HASENCLEVER, L. Economia industrial: fundamentos teóricos e práticas no Brasil. Rio de Janeiro: Campus, 2002. 680p.

MALAFAIA G.C. et al. Atitudes de coordenação de produtores rurais na cadeia bovina: o caso do CITE 120. Organizações Rurais \& Agroindustriais, v.11, n.3, p.393-406, 2009. Disponível em: $<$ http://revista.dae.ufla.br/index.php/ora/article/viewArticle/41>. Acesso em: 15 mar. 2010. 
MANGABEIRA, J.A.C. et al. Tipificação de sistemas de produção rural: a abordagem da análise de correspondência múltipla em Machadinho d'Oeste-RO. Campinas: EMBRAPA, 2002. 26p. (Circular Técnica, 8).

OAIGEN, R.P.; BARCELLOS, J.O.J. Gerenciamento e custo de produção. In: MOURA, J.A. et al. (Eds). Programa de atualização em medicina veterinária. Porto Alegre: ARTMED, 2008. p.51-88.

SAAB, M.S.B.L.M. et al. O desafio da coordenação e seus impactos sobre a competitividade de cadeias e sistemas agroindustriais. Revista Brasileira de Zootecnia, v.38, supl. esp., p.412-422, 2009. Disponível em: <http://www.scielo.br/scielo.php?pid=S151635982009001300041\&script=sci_arttext $>$. Acesso em: 18 jan. 2010. doi: 10.1590/S1516-35982009001300041.

SEBRAE/SENAR/FARSUL (SERVIÇO BRASILEIRO DE APOIO ÀS MICRO E PEQUENAS EMPRESAS / SERVIÇO
NACIONAL DE APRENDIZAGEM RURAL / FEDERAÇÃO DAAGRICULTURA DO ESTADO DO RIO GRANDE DO SUL). Diagnóstico de sistemas de produção de bovinos de corte do Estado do Rio Grande do Sul. Porto Alegre, 2005. 257p.

SILVA, C.A.; BATALHA, M.O. Competitividade em sistemas agroindustriais: metodologia e estudo de caso. In: WORKSHOP BRASILEIRO DE GESTÃO DE SISTEMAS AGROALIMENTARES, 2., 1999, Ribeirão Preto, SP. Anais... Ribeirão Preto: PENSA/FEA/USP, 1999. p.9-20.

SILVA, C.L. Fatores determinantes da competitividade internacional da indústria de papel de imprimir e escrever sob ótica da cadeia de valor. Revista Brasileira de Gestão de Negócios, v.6, n.14, p.42-59, 2004.

VAN DUREN, E. et al. Assessing the competitiveness of Canada's agrifood industry. Canadian Journal of Agricultural Economics, v.39, p.727-738, 1991. 